\title{
"LAW AND LEARNING THEORY" THROUGH THE LOOKING GLASS OF LEGAL THEORY*
}

\author{
BY HESSEL E. YNTEMA †
}

I.

IN the ferment of notions that has characterized thinking about law in the United States during the past generation, argument has streamed chiefly from two types of attitudes-the fundamentalist and the scientific. On the one hand, there has been a clearly discernible and progressive trend to regard law, not as the instrument of personified supernatural intelligence or of self-sufficient moral entities, but as a category of space and time, involving human relations, and subject to human devising, direction, and purpose. This much is the common denominator of the ideas $\longrightarrow$ of law in action versus the law of the books; of law as a means of social control, a phase of social science, a constructive process of social engineering; as the instrumental procedure, the applied discipline, or the behavioristic description of government; and of other like conceptions that have served in recent times as motives for the principal renovatory movements in general legal doctrine. These conceptions represent an effort finally to secularize the department of justice, to subject it to the experiments and hypotheses of science; in a word, by enforcing the principle of the two swords under which the independence of the modern State was achieved, to predict and so to govern man's relations with man on the basis of knowledge comparable to that provided by modern science for man's relations to physical nature.

Whoever have thus entered the hitherto sacred precincts of the temple of justice, intent upon piercing the heavy veils of superstition within, in order that, as they pretend, they may remove the scales from the eyes of the blind goddess, have seemed to many acolytes of the temple as barbarous as the medieval barber-surgeons responsible for the science of medicine once did to the doctors of that profession nursed upon Hippocrates. Those who have been indoctrinated in the tough traditions of the professional schools, those too much pained by the slow and halting progress of social science, those, perchance, caught up by the vision of law as the sacred art of distributive justice-or who have accepted as the framework of their analyses of things legal the patterns of philosophies forever dividing mind and matter, form and substance, "is" and "ought," the supernal from the "real,"-necessarily, in defining law, tend to attribute to it the character of hallowed custom, inevitable dialectic, or ultimate truth. To such, law is essentially found not made, declared and not

* Moore and Callahan, Laze and Learning Theory: A Study in Legal Control (1943) 53 YALE L. J. 1.

$\dagger$ Professor of Law, University of Michigan. 
questioned, a religion concerned with the mundane realization of a believed in verity beyond change, time, and space. These, the fundamentalists, of course, have objected to the profaning of the temple by those who come with measure and with microscope in the name of science. The ensuing conflict of ideas has inspired the most vivid and significant developments in the formal legal theory of our times.

Obviously, the lines are not so clearly drawn as the above suggests. One who in youth publicly embraces the cause of science may later espouse a decorous fundamentalism. (Apparently, the conversion to Aristotelian authority may be more sudden; it has occurred in moving from one part of the country to another.) There are always, too, the eclectics, who say that law is too complex to be adumbrated in a single simple analysis, the mugwumps of legal theory, who avoid the solution of the complexity by accepting all plausible solutions. Nay more, the issue between fundamentalism and science in the field of law probably is spurious; the more intelligently the spirit of the great scholars invoked as authority to justify the fundamentalist attitude is sought out, the nearer is the investigative mood of science approached. The very greatness of such as Aristotle or Aquinas is that they strove to attain knowledge, to unite reason and experience. The presumption that what they found is forever enough denies the motive that made them great. And it seems supererogatory to suggest that to seek to describe occurrences with a view to predicting their repetition is neutral with respect to their possible relations to immanent essences. Fundamentalism has no monopoly on faitl; it seels merely to stop inquiry. This suggests that the issue posed by fundamentalists is whether legal doctrine should be satisfied with the notions of some time past, deemed authoritative, or shall seek knowledge more complete and precise.

II.

Laid on the background thus vaguely sketched, the study in legal control reported by Underhill Moore and Charles C. Callahan is of interest as an effort to ascertain and thus to demonstrate whether it is possible to correlate a limited number of segments of experience, containing supposedly legal phenomena, with hypotheses stated in terms of a discipline assumed to be pertinent, namely, experimental psychology. The report sets forth in detail the considerations governing the selection of human behavior to be observed, the conditions under which observations occurred, the units of measurement adopted, and the assumptions made in correlating what was observed; it exhibits the same in tabular and graphic form, and, on the basis of a meticulous analysis, presents a mathematical equation in terms of which the relations between the frequency distributions in the segments of experience compared are stated. Equally exhaustive is the authors' interpretation of the statistical observations by reference to a 
theory of behavior, denominated "Learning Theory," as a means of ascertaining whether the measurements either are or correspond to the "entities" of a general theory of behavior.

For details, the reader is referred to the report itself; it is not proposed in this casual comment to recapitulate the data or to endeavor to restate the succinct interpretation thereof in terms of the "Learning Theory." For the purposes of a review of the matter from the viewpoint of legal theory, it may be assumed that the observations were accurately made and recorded, that the calculations were duly checked, and that the interpretation is logically correct. In other words, the study may be taken as a demonstration that, in a limited sample of experience, the incidence of particular instances of legal regulation upon human conduct is susceptible of measurement and statement in quantitative form, the units of which represent a general theory of human behavior. The question presently pertinent from the viewpoint of legal theory is the significance of stuch a demonstration.

It is no little thing, as any who have essayed to report social events in statistical form will be aware, to construct an investigation as exactly and to report the findings as candidly as the authors have done. Be the results positive, negative, or indifferent, their report is a lesson in method, pect1liarly praiseworthy in view of the prodigious fumbling that has sometimes passed for statistics about law. In particular, it exhibits the difficulties encountered in reducing observations of relatively simple social phenomena to a comparable statistical basis and in segregating and formulating the influence of an administrative regulation or other "artifact" as an index, quantitatively stated, of the effectiveness of legal control. But there is more than this. A showing that in a limited number of observed segments of human conduct variations occur, coincidentally with the absence or presence of acts or signs designed to regulate such conduct, and that such variations can be measured and interpreted to exhibit regularities (all of which may be fairly assumed for the present study), is an indication that human conduct generally in the presence of legal regulation is subject to regular and measurable variations. Just as the dissection of a normal human heart teaches the structure of all human hearts, so this study portends that examination of other phenomena of control of social conduct by law will reveal analogous measurable regularities. Given the existence of a universe, as contrasted with a chaos, of human conduct, the typical sample must reflect the structure of all comparable experience.

The obvious implications of so much are perhaps worth a remark. It has the significance that human conduct as the object of legal regulations and the effects of such regulations on such conduct are susceptible of scientific observation and measurement. It implies that, for scientific inquiry, it may be presumed that the acts or omissions of human beings 
may be conditioned by legal regulations, that human conduct need not be regarded for the purpose as essentially involving the exercise of freewill. In other words, the study supports the tenability of the behavioristic hypothesis as a basis for the statement of the effects of legal control. This seems trite; it is nerertheless important, chiefly on account of the frequent assertions that, by its very normative nature directed to human beings with freedom of choice, law necessarily is incalculable in its effects. The evidence adduced by the authors suggests that it is feasible in a scientific inquiry of the kind to ignore free-will (though doubtless not always variations of intelligence) as a factor to be taken into account. Assuming it to be characteristic of human beings that they are born free, the universal fact seems as immaterial to the purpose, as the circumstance that they are bipeds. For statistical purposes, such common elements are not significant, since they do not differentiate (a blind-spot in statistical technique).

The foregoing general observations are especially motivated by the slow-down that has taken place in the field of jurisprudence during the past fifteen years. Notably, there has been relaxation in the prosecution of the efforts, which looked so promising in the twenties, to extend in scope and in depth the fragmentary scientific knowledge of legal phenomena. Then, many things were hopefully burgeoning: the Restatement was riding on a crescent moon; the cross-fertilization of law with other social sciences was a commonly accepted objective of reforms in legal education; and there was inaugurated a promising succession of efforts to increase the available information concerning the legal process, both through the collection of judicial statistics and to a minor degree by historical and comparative research. These movements were in part implemented by the creation of research institutions, which in the then favorable atmosphere promised a significant renascence of legal science in this country. In retrospect, it seems unfortunate that those happy days were closed by the spurious rivalry with which so-called realistic jurisprudence challenged the then more respectable sociological brand; the dispute divided energies that could not be spared. For in the thirties came the deluge: the depression, the New Deal, and latterly, the war. In consequence, funds available for legal research evaporated or were claimed for more imperious, immediate purposes, with the result that such hopeful ventures as the Institute of Law at The Hopkins, with which the writer was connected, and others of the kind had to be abandoned or materially reduced. More fateful still, the ranks of legal scholarship have been seriously depleted; too many who would otherwise have been destined to lead the procession of jurisprudence have been impressed or seduced into public service, never to return, and for the time being the manhood of the country has necessarily gone to war. 
But, if there be one thing clear in these stirring and disturbing times, it is that there was never an occasion when sound legal statesmanship was more desperately needed, never a juncture in the history of this country when reliable information on law has been more exigently required. A mere glance at the problems unresolved by the administrative experimentation of the past decade and the even more momentous issues being generated by the current hostilities, will suffice to dispel any doubt of the need. In the face of impending social reconstuction, we have too largely to improvise or, what is worse, to make a virtue of opportunism. For these reasons, it is genuinely encouraging that the authors have had the singleness of purpose to pursue, and Yale University the farsightedness to support through the Institute of Human Relations, research directed to the fundamental aspects of legal control. As the authors have stated, until through such research precise knowledge of the effects of law on human behavior is made available, "any discussion of the relative desirability of alternative social ends which may be achieved by law is largely day-dreaming and any discussion of the 'engineering' methods by which law may be used to achieve those ends is largely futile." 1 In days when jurisprudence has been so largely devoted to speculative literature, the assertion is pertinent. It is worthy of the liberal tradition, since Hohfeld, of the University in which the spirit of legal science has made its home.

III.

Yet tribute, however deserved, to the objectives of the study and passing recognition of its methodological significance are not enough. Even in the nebulous looking-glass of legal theory, through which this review is essaying to peer, certain extrinsic features in the background of the study are to be noticed. For one thing, as it may be suspected the authors would be the first to concede, granting that the study serves as a valid demonstration of the possibility of describing the effects of legal regulation in a quantitative formula, the results, even for the particular type of human behavior observed, need to be verified and refined by analogous observations of similar behavior in other times and places. To extend the metaphor: dissection of a normal human heart indicates the structure of all human hearts, but it is necessary to take apart a sufficient number of human hearts to be assured that the one dissected is normal. And it may be added, in view of the nice problems of construction presented by the observed data, the results of the study for this reason too should serve to stimulate rather than to foreclose further research respecting the effects of ordinances, tagging, and other modes of regulation on parking behavior.

1. See article by Moore and Callahan in My Purosophy of LAw (1941) 201, at 206-07. 
There are, however, broader and more trenchant considerations, involving the problem of the directions in which the limited resources available for legal research should be expended. The reader will perhaps permit the issue to be pointed up by reference to an early episode in the relations between the reviewer and the principal author of the study under consideration-perhaps the first intellectual contact. It was in the halcyon days before the deluge, and the reviewer had painstakingly prepared, as he thought, an exhaustive if not exhausting, account of the interesting morphological history of the Lex Murdrorum from the Norman Con. quest to date, which was duly published. Whereupon, Underhill Mnore observed his regret that so much effort had been expended on such a subject. The validity of the observation of course depended on the point of view, but it impressed the reviewer, then a callow undergraduate on the Columbia faculty, as going to a proper point, germane to any legal research.

The point suggests a series of questions. First, the study specifically is directed to the incidence of legal artifacts upon parking behavior. Is this the significant aspect of what may be generically termed the parking problem? Second, the area of human behavior selected for examination is characterized as that of the "normal," as contrasted with the abnormal. the supposedly less repetitive area of litigation. The issue here, among other things, is as to the general applicability in the study of legal phenomena of quantitative, as distinguished from qualitative, techniques. Third, the study hypothesizes conformity of human behavior as the criterion of legal propositions. The presuppositions of this choice, expressly indicated by the authors, apparently involve the exclusive significance of this mode of evaluation and incidentally controvert the often emphasized normative implications of legal propositions. Obviously, these three are difficult questions; in view of the scarcity of available energies for legal research, they have to be faced, however, even though they may not be adequately answered. What follows is, therefore, intended as prorocative suggestion, not necessarily criticism.

The first issue faces the objective of regimentation. Is man and his behavior made to be regimented or is regimentation to be adjusted to behavior? Implicit in this is the perennial conflict between the conception of paternalistic or socialist autocracy and the more modern notion that to govern is to serve. Both notions seem to be consistent with the wavering concepts of democracy; political organizations supposedly democratic at least have acted on the theories both that the individual is subject to the State and that the State and its officers must answer to the individual. With more particular reference to the matter in hand, it might be worth turning the problem upside down and to inquire, in setting the perspective of a study of the relation between legal artifacts and parking behavior, not whether behavior conforms to artifact but whether artifact conforms 
to behavior. If it be assumed that the objective mediately sought is not to ascertain what modes of control are most effective in preventing undesired parking, but what modes of control provide the most effective use of parking facilities, the immediate object of the study would necessarily invite correlation with a congeries of further intriguing problems concerning the appropriate adaptation of administrative techniques to parking needs. What is proposed, in other words, is that, on a behavioristic hypothesis, the study faces a twofold problem. If the effectiveness of the artifact, itself administrative behavior, is to be determined by the behavior to which it forms, in the authors' language, a sign, then, it is submitted, the criterion of the sign is the behavior assumed as the norm. It is not intimated that this is necessarily inconsistent with the stated premises of the study, except perhaps in the form of statement.

The second issue is presumably more controversial. It may be focused by the following exaggerated inquiry: If there were to be in the next ten years in the United States only one quantitative study of legal phenomena, what should it take as subject matter, the "normal" area of non-litigious behavior or behavior connected with actual or possible litigation? The sensible answer is, of course, both; but the present study is based on certain presuppositions which should be noted. Primarily, we are again faced with the premise that the object of law is to control behavior. Even if this be true, it does not exclude the possibility that there may be other and perhaps more rudimentary purposes in the legal process. It may, for example, be a purpose of law to keep the peace among other things by providing a pacific procedure for settling disputes in a manner that will soothe conflicting interests and passions. As to this possibility, it may be briefly remarked that the hypothesis fits the primitive beginnings of embryonic political organization and in addition serves to explain somewhat the mystery of jurisprudence as a technique of persuasion and assuagement. It has been only latterly that the State has preempted the function of executive regulation with which the present study is concerned.

But if litigious behavior, deemed to be atypical, be admitted as a suitable object of scientific description, there appear at once difficulties in the employment of quantitative techniques. Shall the normal form the standard for the abnormal? If the area of litigation be described as of itself, does one start from the client, the advocate, the expert witness, the judge, or perhaps the intuitive bystander or jury? Moreover, is it reasonable to suppose that atypical phenomena-that is, by reference to non-litigious events-will present sufficient regularities to justify quantitative researches? Here is the rub. True, statistical studies have been projected on the supposition that, given a sufficient base, the phenomena of litigation will reflect significant trends or stresses in social structure or function, as well as permit the formulation of regularities within the adjudicative process itself. But such studies are perforce of the shotgun type; and, 
when a precise examination of individual conflicts that have festered to the threshold of litigation is to be made, there is often a case for qualitative rather than quantitative modes of investigation.

In other words, it would seem that the election of a quantitative method influences the choice of the matter to be examined and the problem to be resolved. To use statistics, there must be observable repetitious events; and, what is not always understood, the terms in which such events may be analyzed must be relatively simple. If the phenomena inquired into are unusual or if they are not resolved into a limited number of factors, quantitative formulation is either of minimal significance or cumbrous.

Moreover, economy in the utilization of research energies here too has bearing. Quantitative methods may be costly in time and effort relative to what they may reasonably be expected to accomplish. Meanwhile, the process of social change rushes on, and there are new issues to be resolved. All this counsels that, while it is most necessary to secure precise, quantitative knowledge where it is feasible and appropriate, it is also expedient to employ more expeditious, if less exact, tecluniques of objective inquiry where they are indicated. To invoke once more our medical simile; the structure of the heart is not distinctively a quantitative problem.

This drives on to the third basic and even more controversial issue that it is proposed to touch. Is it legitimate to study legal propositions without reference to their conformity to or effect upon behavior? Remarking on the common failure to do so, the authors attribute the custom to the presupposition, inter alia, that the degree of conformity of conduct to legal rule, if not complete, is known, on which presupposition they remark:

"Entertaining it leads natural-law and analytical jurists to restrict the study of law to dialectic; historical jurists to the art of writing either the history of a literature of legal propositions or the history of a larger fragment of culture; sociological jurists to speculation upon the more remote consequences of propositions of law, speculation upon the effect of the supposedly known but in fact unknown quantity and degree of conformity to the proposition upon behavior which is not prescribed or proscribed in the proposition; and 'realists' to random behavior."2

The challenge to these attitudes presented by the authors in the present investigation is, we have already avowed, salutary; indeed, as may appear here and elsewhere, it fully enlists the reviewer's enthusiasm. But insofar as it implies (if it does) that there is a single, quantitative technique for legal research, it too must be challenged. That a researcher should publish his enthusiasm for the technique that he has carefully refined as unique and exclusive is admirable, but it does not preclude other enthusiasms. In the present case, to ask that legal research be confined to quantitative

2. P. 1 . 
techniques, deemed however intrinsically desirable is like postponing horseracing to the invention of pari-mutuels.

The fact is that there is no hierarchy of methods; technique must fit the problem to be resolved, even in the ascertainment and formulation of truth. As the reviewer once took occasion to suggest, ${ }^{3}$ there are four distinctive modes of obtaining data to verify hypotheses suggested by observation of legal phenomena-experiment, quantitative observation, comparison in time, and in place. Other techniques of objective inquiry may perhaps be distinguished; e.g., qualitative observation of types and combinations are of course common. The present study, for instance, supplements the observation of frequencies in behavior with ingeniously controlled experimentation. Of these modes of verification, it has been the reviewer's judgment, predicated chiefly upon the limited, formal, and provincial scope of knowledge about law, that the most promising avenues of inquiry to extend and to enrich this knowledge are: first, exploration of the operational or effective aspects of law, including especially studies of the kind here in view; and, second, comparison of institutions in different legal cultures, involving, for reasons set forth in the discussion referred to, ${ }^{4}$ both historical individualization and reference to comparable phenomena in two or more such cultures. Although in comparative legal research recourse to quantitative measurements is, of course, dusirable where pertinent, it is so frequently impossible or infeasible to ascertain the existent frequencies in the phenomena that the admission of this type of investigation supposes that it may be valid in the absence of quantita. tive measurement. This presents the crux of the issue: Must legal science always think in numbers and frequencies?

On this it might be observed, by way of persiflage, that the normal behavior of legal discourse is, as the authors duly charge, non-quantitative; if frequency alone be persuasive, this is perhaps an argument. More substantially, it may be remarked that the authors propose that law, characterized as an "artifact," should be evaluated in terms of its correspondence to the behavior that is its object, whereas the present suggestion is that it is also appropriate in suitable cases to compare artifact with other like artifacts differentiated in time or place. Legal artifacts being after all but behavior, this supposes that comparison of behavior in comparable segments of experience may yield significant inferences. Indeed, this is supposed in the present study, but the segments selected are not sufficiently disparate in time or place to ensure that conditions peculiar to New Haven have been accounted for. The results would seem more generally persuasive if the observed ratios of conformity to legal regulation were shown to correspond to ratios representing analogous conformities, say,

3. See YNTEAra, Roman Law as the Basis of Comparative Law in 2 Law: A CENTURY OF PROGRESS (1937) 346, 360-64.

4. Ibid. 
in the horse and buggy days, or in Cairo. The comparative method serves in a measure to factor out the local variations, which quantitative analysis of a given unity of behavior is unable to do.

The proposition, stated above, that legal artifacts, including formal legal propositions, may be studied as a species of behavior, needs a concluding word, in view of the authors' insistence that such propositions do not always correspond to what they are presupposed to signify. Perhaps here is the central difficulty. Law, as the authors define it, consists of signs intended to influence conduct. In his intriguing treatment of this matter, Thurman Arnold has stressed, but perhaps not entirely appreciated, the symbolic, inspirational nature of law. It reflects, as he has so trenchantly noted, the confused and contradictory aspirations of humanity, the ideals of time and place, a world of make-believe, which is nevertheless existent and real. It needs to be added that, as suggested above, one of the purposes of law is to keep the peace, to resolve conflicts with the least dissatisfaction, and therefore it may be sometimes more important, at least in the sphere of adjudication, that the rule should seem just than that it be always observed. Whether or not this be equally true of administrative regulation, regarded from the viewpoint of the executive, so much must be accorded to the common notion that law is normative; it is a perverse inference that legal science has an equally ideological function. $^{5}$

To repeat, the foregoing are intended as but provocative suggestions, not criticism of a most instructive investigation. When the lovelorn swain places a pearl on the finger of his inamorata, it has to have a setting. But it is still a pearl.

5. See the reviewer's discussion in The Ratimal Basis of Leyal Scicnec (1931) 31 CoL. L. Rev. 925, 942 et seq. 